\title{
Effective Fast Response Smart Stick for Blind People
}

\author{
Ayat Nada, Samia Mashali, Mahmoud A. Fakhr, and Ahmed F. Seddik
}

\begin{abstract}
Visually impaired people find difficulties detecting obstacles in front of them, during walking in the street, which makes it dangerous. The smart stick comes as a proposed solution to enable them to identify the world around. In this paper we propose a solution, represented in a smart stick with infrared sensor to detect stair-cases and pair of ultrasonic sensor to detect any other obstacles in front of the user, within a range of four meters. Moreover, another sensor is placed at the bottom of the stick for the sake of avoiding puddles. Speech warning messages and the vibration motor are activated when any obstacle is detected. This proposed system uses the microcontroller $18 F 46 K 80$ embedded system, vibration motor and ISD1932 flash memory. The stick is capable of detecting all obstacles in the range 4 meter during $39 \mathrm{~ms}$ and gives a suitable respect message empowering blind to move twice his normal speed because she/he feels safe. The smart stick is of low cost, fast response, low power consumption, light weight and ability to fold.
\end{abstract}

Keywords - Infrared Sensor, Ultrasonic Sensor, Electronic Travel Aids (ETAs), Visually impaired, Blind Navigation.

\section{Introduction}

Visually impaired people are the people who can't identify smallest detail with healthy eyes. Those who have the visual acuity of $6 / 60$ or the horizontal extent of the visual field with both eyes open less than or equal to 20 degrees, these people are considered blind [1]. Such people are in need of aiding devices for blindness related disabilities. As described in [2] $10 \%$ of blind have no usable eyesight at all to help them move around independently and safely. The electronic aiding devices are designed to solve such issue.

To record information about the obstacles presence in a road, active or passive sensors can be used. In case of a passive sensor, the sensor just receives a signal. It detects the reflected, emitted or transmitted electro-magnetic radiation provided by natural energy sources. In case of using an active sensor, the sensor emits a signal and receives a distorted version of the reflected signal. It detects reflected responses from objects irradiated with artificially generated energy sources. These kind of active sensors are capable of sensing and detecting far and near obstacles. In addition, it determines an accurate measurement of the distance between the blind and the obstacle. Overall, in the obstacle detection domain,

Ayat Nada ${ }^{1}$, Samia Mashelly ${ }^{2}$, Mahmoud A. Fakhr ${ }^{3}$

Computers and Systems, Electronics Research Institute.

Giza, Egypt.

Ahmed F. Seddik

Biomedical Engineering, Faculty of Engineering, Helwan University. Cairo, Egypt. four different types of active sensors may be used: infrared, laser, ultrasonic, in addition to radar sensors.

Bat K Sonar [3], Smart Cane [4], Smart vision [5], Guide Cane [6], use ultrasonic sensors or laser sensors to detect obstacles in front of blind by transmitting the wave and reception of reflected waves. It produces either an audio or vibration in response to detected obstacles to warn blind. Systems like vOICe [7], SoundView [8], SVETA [9] and CASBLIP [10], use single camera or stereo video cameras mounted on a wearable device to capture images. These captured images are re-sized, processed further and converted to speech, audio, musical sounds or vibrations. In such systems, the frequency of warning sound signal is correlated with the orientation of pixels. Some advanced systems use Global Positioning System (GPS) integration with the main system. It's also noteworthy that GPS receiver is useful for understanding the current location of the subject and nearby landmarks. Some solutions are already available in the market such as: UltraCanne [11], Isonic [12], and Teletact [13] and others [14]. These products help blind people by collecting information through sensors and then transmitting recommendations through vibration or sound message to the user.

These solutions still have many disadvantages for example;

They can't detect obstructions that are hidden but very dangerous for the blind such as downward stairs, holes etc. Usually, the feedback information comes out as either vibration or sound signals. Thus, these systems communicate their recommendations to the user through sound or frequency vibration.

Consequently, training is then necessary to help the user understand the signals and to react to them in real time. However, such training is sometimes more expensive than the product itself. Therefore, users can't afford it [15]. Otherwise, the information is transmitted as a sound it may be embarrassing for the blind person in public.

In our work we tried to overcome some of disadvantage:

- We designed stick to detect obstacles and its able to recognize and speak aloud the upward and downward stairs or puddles as shown in Fig. 1.

- The training of our product isn't as expensive as training in other product. Our training is just description of stick component and usage position.

- We use two facilities to transmit information to the blind. We integrated vibration motor in the hand of stick and speech warning message kit that preserving it's natural dimension to keep it user friendly.

- With aid of earphone the speech warning message kit is able to speak aloud warning message to the blind 
Proc. of the Second Intl. Conf. on Advances in Bio-Informatics and Environmental Engineering - ICABEE 2015

Copyright $\odot$ Institute of Research Engineers and Doctors, USA .All rights reserved.

ISBN: 978-1-63248-043-9 doi: 10.15224/ 978-1-63248-043-9-29

instead of incomprehensible sound and public embarrassment.

- We achieved very fast response time calculated as 39 $\mathrm{ms}$ in average distance $\leq 400 \mathrm{~cm}$ before hitting the obstacles.

- Regarding ease of use and independence of blind we added a facility of easy search to the stick in the form of remote (location or positioning of the stick).

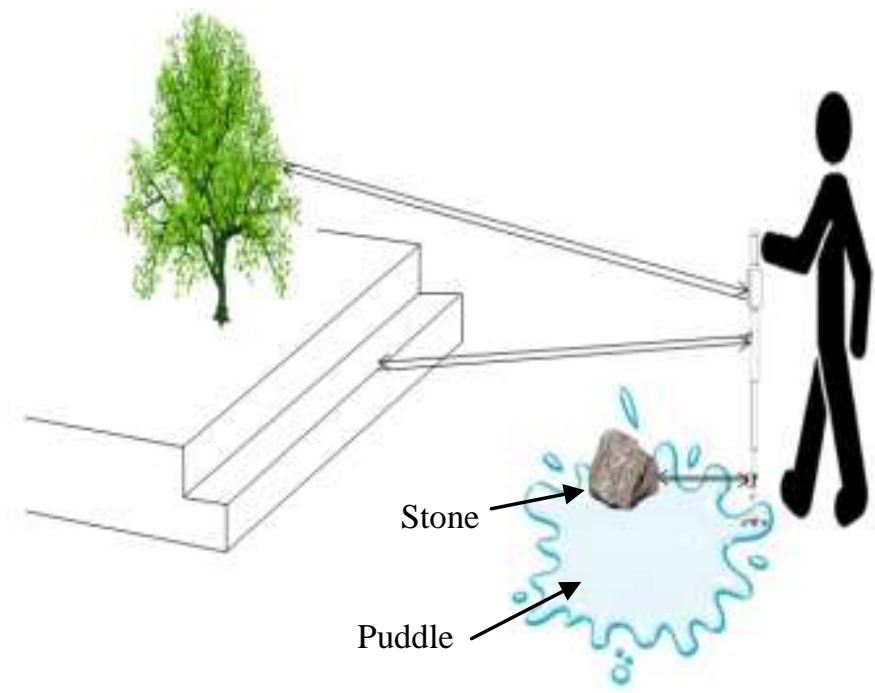

Figure 1. Smart stick detects obstacles in front of the blind.

\section{Proposed System}

We have many reasons to design smart stick for blind; firstly, the blind to feel free, isn't surrounded by wires as in belt and its content. Secondly, is easy to use because it is familiar and affordable. Thirdly, to be able to detect obstacles that exist on the ground (this is not available in glasses), which he walks indoor and outdoor is faced by obstacles such as stairs, puddles and sidewalks.

The smart stick, as shown in Fig. 2, is basically an embedded system integrating the following: pair of ultrasonic sensor to detect obstacles in front of the blind from ground level height to head level height in the range of $400 \mathrm{~cm}$ a head, infrared sensor to detect upward and downward stairs. Ultrasonic sensors and infrared sensor collect real time data and send it to $18 \mathrm{~F} 46 \mathrm{~K} 80$ microcontroller. After processing this data, the microcontroller actives the motor to vibrate and invokes the right speech warning message stored in ISD 1932 through an earphone. Water sensor to detect water spreads, rechargeable battery to power the circuits.
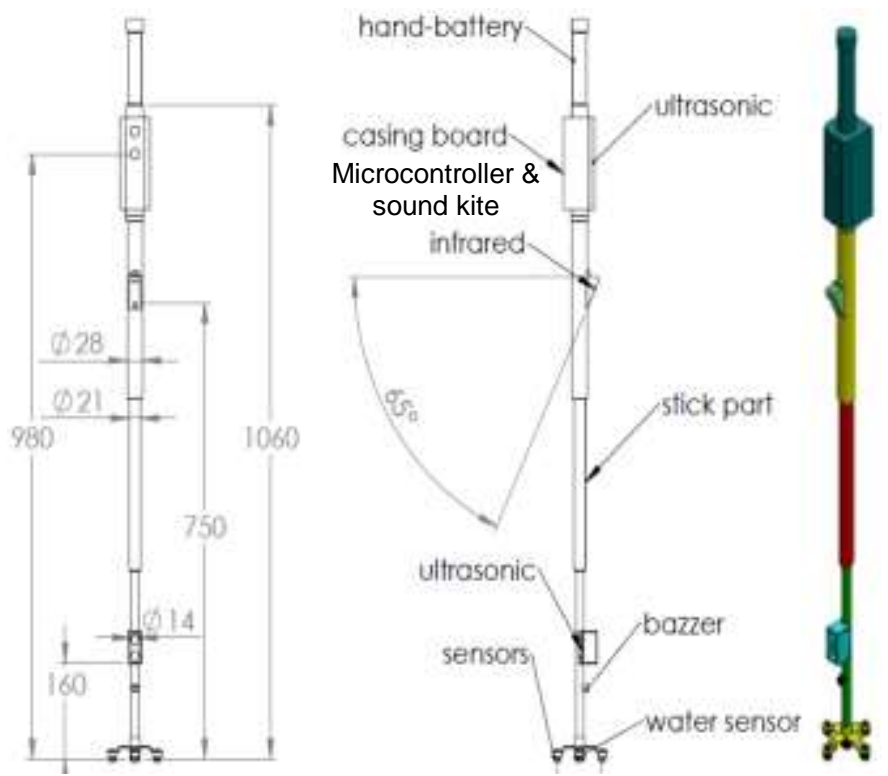

Figure 2. Design of the smart stick Smart stick detects obstacles in front of the blind.

Fig. 3 shows the proposed system. The following subsections will discuss each block in more details.

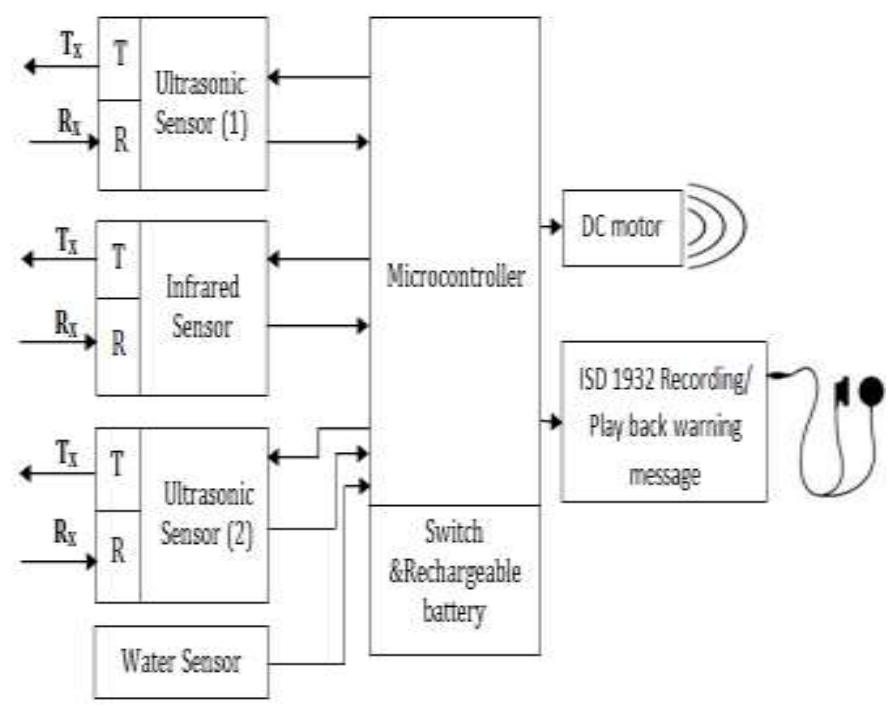

Figure 3. Block diagram of electronic smart stick.

\section{A. Sensors}

The selection process of appropriate sensor depends on several factors such as, cost, atmospheric condition, kind of obstacle to be detected, detection range, and the desired precision of measurements collected information and its transmission frequency as shown in Table I.

We used a combination of 2 types of sensors infrared and ultrasonic for the following reasons:

a) Infrared sensor recognize small obstacle but with less accuracy than laser sensors. However using laser sensor is costly which contradicts our aim in obtaining affordable aiding devices. They perform almost the same within 2 meter. 
b) Ultrasonic sensor work well for close obstacles unlike laser one, when an object is so close the laser sensor (less than $15 \mathrm{~cm}$ ) can't get an accurate reading. Moreover, it should be noted that radar sensors can easily detect near and far obstacles with equal perform once, but their medium accuracy doesn't allow them detecting small obstacles.

TABLE I.

General Characteristic OF SOME ACTIVE Sensors

\begin{tabular}{|c|c|c|c|c|}
\hline & Laser & Infrared & Radar & Ultrasound \\
\hline Principle & $\begin{array}{l}\text { Transmission } \\
\text { and reception } \\
\text { of light wave }\end{array}$ & $\begin{array}{l}\text { Transmissi } \\
\text { on and } \\
\text { reception of } \\
\text { pulse of IR } \\
\text { light }\end{array}$ & $\begin{array}{l}\text { Transmission } \\
\text { and reception } \\
\text { of microwave }\end{array}$ & $\begin{array}{l}\text { Transmissi } \\
\text { on and } \\
\text { reception of } \\
\text { acoustic } \\
\text { waves }\end{array}$ \\
\hline Range & $\begin{array}{c}\text { SLR: } 15 \mathrm{~cm} \text { to } \\
120 \mathrm{~cm} \\
\text { LLR: about } \\
10-50 \mathrm{~m}\end{array}$ & $\begin{array}{c}\text { From } 20 \\
\mathrm{~cm} \text { to } 150 \\
\mathrm{~cm}\end{array}$ & $\begin{array}{c}\text { about } 150- \\
200 \mathrm{~m}\end{array}$ & $\begin{array}{l}\text { From } 3 \mathrm{~cm} \\
\text { to } 10 \mathrm{~m}\end{array}$ \\
\hline $\begin{array}{l}\text { Beam } \\
\text { width }\end{array}$ & narrow & fairly thin & $\begin{array}{c}\text { Depended on } \\
\text { size of } \\
\text { antenna } \\
\end{array}$ & wide \\
\hline $\begin{array}{l}\text { Atmosph } \\
\text { eric } \\
\text { condition }\end{array}$ & affected & affected & Affected & $\begin{array}{c}\text { Not } \\
\text { affected }\end{array}$ \\
\hline Cost & Very high & Low & High & Low \\
\hline
\end{tabular}

SLR: short laser range, LLR: Long laser range

Infrared sensor chosen has a detection range distance that goes from 20 to $200 \mathrm{~cm}$, a resolution of $0.5 \mathrm{~cm}$, a frequency of $26.3 \mathrm{~Hz}$ and an analogical output that goes from 0 to $5 \mathrm{~V}$.

Ultrasonic sensor used $40 \mathrm{kHz}$ transmission signal. The 40 $\mathrm{kHz}$ frequency is produced by a transmission sensor of twocentimeter diameter; it can generate 2.4644 beams of narrowness. This is a reasonable size to be installed in the stick.

We use the infrared sensor to detect upward and downward stairs because the sensor spot is roughly $6 \mathrm{~cm}$. This feature enables the user to identify precisely, any kind of stairs in front of him.

We use a pair of ultrasonic sensor. An upper one at a height $90 \mathrm{~cm}$ to detect upper obstacles and another sensor at a height $30 \mathrm{~cm}$ to detect low obstacles.

Detection using ultrasonic sensor is based on two factors:

- Time of flight (TOF), the amount of delay between the emission of a sound and the arrival of an echo depending on the distance of an obstacle, which is directly proportional to the distance.

- Beam size: Obstacle size is depending on amount of reflected wave. Obstacles whose dimensions are larger than the beam size, all of the sound waves will be reflected to receiver. If the obstacle size small as compared to the beam size, the part of the ultrasonic sound wave will be reflected to the receiver and the rest will be lost as shown in Fig. 4.

The speed at which sound travels depends on the medium it passes through. Broadly, the speed of sound is proportional to the square root of the ratio between the stiffness of the medium and its density. The speed of sound also changes with the atmospheric conditions. All obstacles reflect some part of the wave through. The amplitude of the wave reflected is relatively proportional to how much available surface there is on the obstacle, concerning coherent reflection. Also, surface area, shape and orientation, are major factors contributing to the strength of the reflected signal.

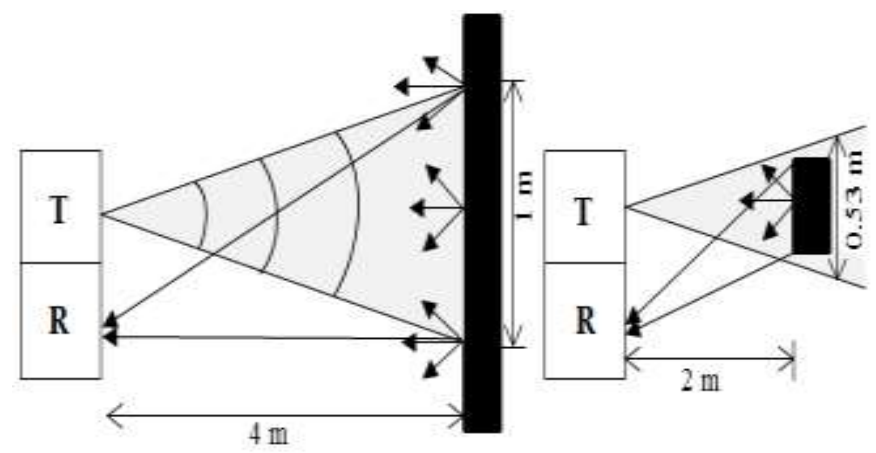

Figure 4. Small and large obstacle detection.

\section{B. PIC Microcontroller $18 F 46 K 80$}

Researchers in $[17,18]$ used the microcontroller 16F877A. It has 3 disadvantages (1) it has no internal oscillator so we will need an external crystal as a clock source. (2) It has memory 256 bytes of EEPROM. (3) It has 8 channels of 10-bit Analog-to-Digital (A/D) converter.

The microcontroller 18F46k80 (MCU) [16] used to control the embedded system. The PIC microcontroller we used is simplified computer with a processor, memory and support for peripherals. The PIC simply runs the program in its memory when it is turned on and it doesn't have an operating system. It has $200 \mathrm{~ns}$ instruction execution, 1024 bytes of EEPROM data memory, self-programming, an 2 ICD, 2 Comparators, 11 channels of 10-bit Analog-to-Digital (A/D) converter, 1 capture/ compare/ PWM functions, a synchronous serial port that can be configured as either 3-wire SPI or 1-12wire C bus, a USART, and a Parallel Slave Port. Regulator LM7805 used to regulate the volt to $+5 \mathrm{~V}$ which input to microcontroller if the input voltage exceeds $+5 \mathrm{~V}$.

As shown in Fig. 5. When the MCU is started, it produces a $40 \mathrm{kHz}$ wave with the duration of $300 \mu \mathrm{s}$. It generates the pulse that will drive the ultrasonic emitter. After sending the pulse, the ADC of MC will read and convert the received wave from each ultrasonic receiver into a digital form. If the ultrasonic sensor received the signal, MC will calculate the distance. If the infrared sensor received the signal, MC will calculate average of the signal shape and amplitude. Accordingly, MC invokes the right speech warning message through an earphone. The proposed system was simulated using Proteus software (Simulation Program) [19] as shown in Fig. 6. The program code was written using $\mathrm{C}$ language. 
Proc. of the Second Intl. Conf. on Advances in Bio-Informatics and Environmental Engineering - ICABEE 2015 Copyright $\odot$ Institute of Research Engineers and Doctors, USA .All rights reserved.

ISBN: 978-1-63248-043-9 doi: 10.15224/ 978-1-63248-043-9-29

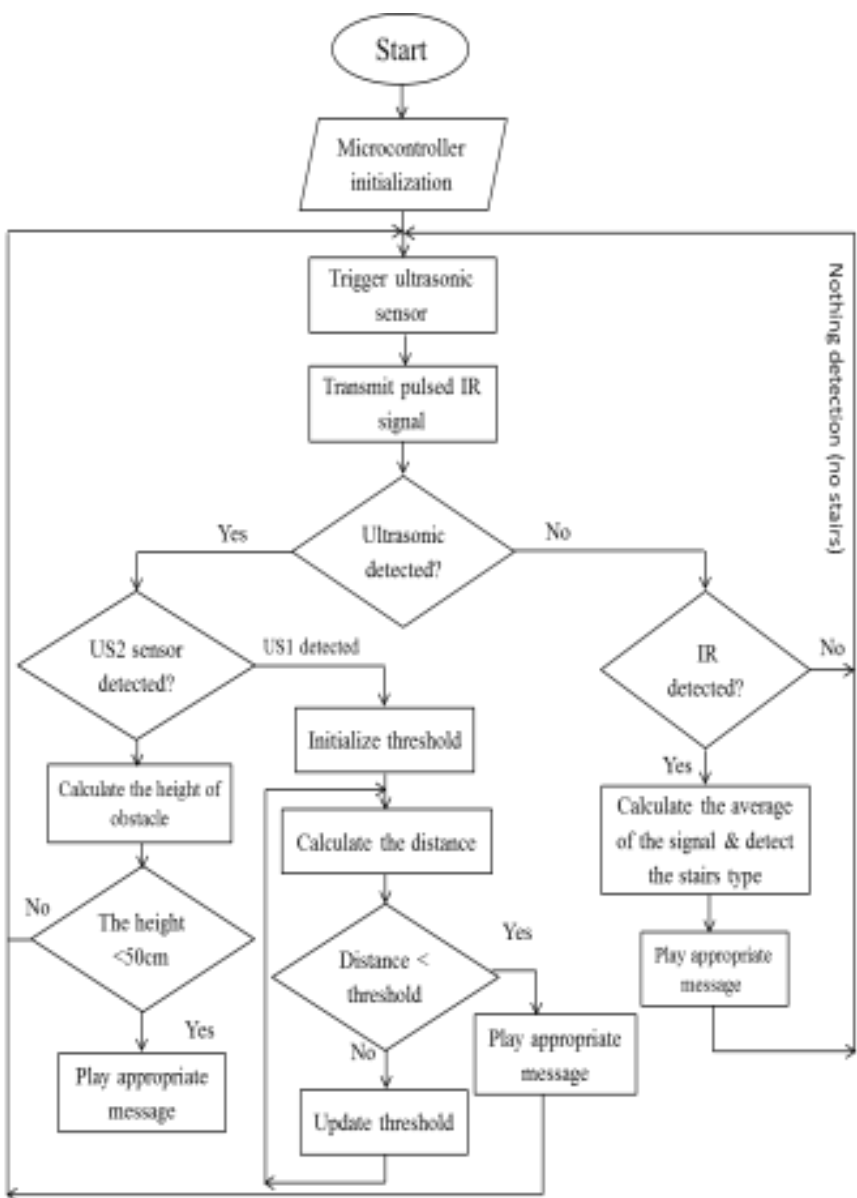

Figure 5. Flowchart of the proposed system.

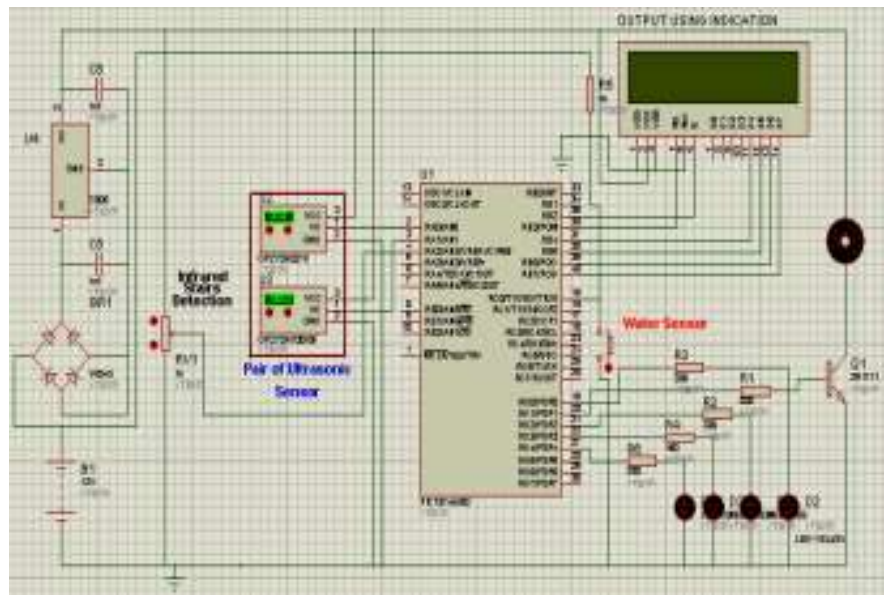

Figure 6. The whole circuit design using Proteus simulation.

\section{Speech Warning Message}

Many researchers $[5,6,14]$ used vibration array or buzzer based audio frequency clips for announcing any detection of obstacles. The proposed stick uses pre-recorded speech messages for conveying any detection of obstacle. It uses ISD1932 [20] circuit that contains a multiple-message recording and playback device. This circuit can record up to 64 seconds per message. It includes microphone inputs and speaker outputs as shown in Fig. 7.

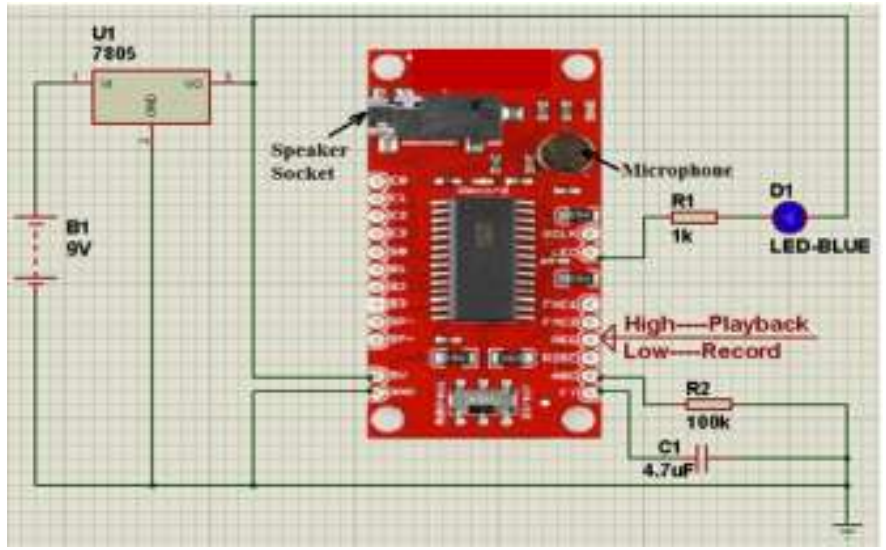

Figure 7. Voice record circuit in direct mode

In the proposed system, we have recorded sixteen different speech warning messages depending on the distanceas to alert the user. We have added a capacitor of $4.7 \mu \mathrm{F}$ and an external resistor of $100 \mathrm{~K} \Omega$ necessary to decrease recording duration to $21.2 \mathrm{sec}$ per message.

\section{Water Sensor}

Water sensors available are used to detect water levels inside tanks and very expensive. Our objective is to detect water existence regardless its level. So we used a costless alternative. Two wire probes are shown in Fig. 8; they fit at the bottom of the stick to sense obstacle like water pits, puddles and water spread. Once wires touch water, the circuit is shorted, this interrupts the microcontroller, activates the vibration motor and play warning message saying: "Attention there is water".

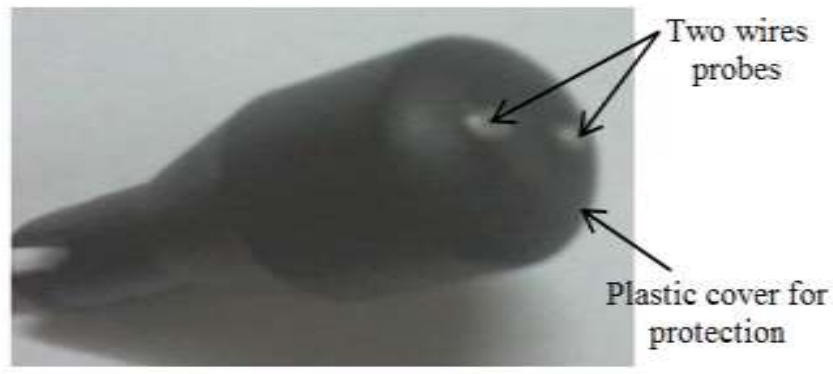

Figure 8. Water sensor

\section{E. Vibration Motor}

This is the type of DC vibration motors used in mobile phones. It requires a voltage supply of $3 \mathrm{~V}$ to $5 \mathrm{~V}$ with current around $125 \mathrm{~mA}$. This type of motors can be programmed to control its speed by using the PWM (Pulse Width Modulation) method. The PWM signal is generated from the TMR2 timer via interrupt control on $\mathrm{RC} 2$ and $\mathrm{RC} 1$ pins to gate this PWM to active the vibration. The diameter of the motor is $0.5 \mathrm{~cm}$ and the thickness is $2.5 \mathrm{~mm}$. 
Proc. of the Second Intl. Conf. on Advances in Bio-Informatics and Environmental Engineering - ICABEE 2015

Copyright $\odot$ Institute of Research Engineers and Doctors, USA .All rights reserved.

ISBN: 978-1-63248-043-9 doi: 10.15224/ 978-1-63248-043-9-29

\section{F. Calling the Stick}

We used FM "frequency modulation" wireless communication to help the blind person to find the stick if it is far from him.

Fig. 9 shows an RF transmitter that generates radio frequency waves in its circuits, and to this 'carrier signal', it adds the information part by modulating the carrier signal. This composite signal (carrier plus information) is then fed to an antenna. An RF receiver receives the signal at the same frequency from the atmosphere, by altering the Electric and Magnetic fields from its own antenna.

The receiver circuits then strip the information part of the signal from the carrier part, and amplify this to a useful level for audio.

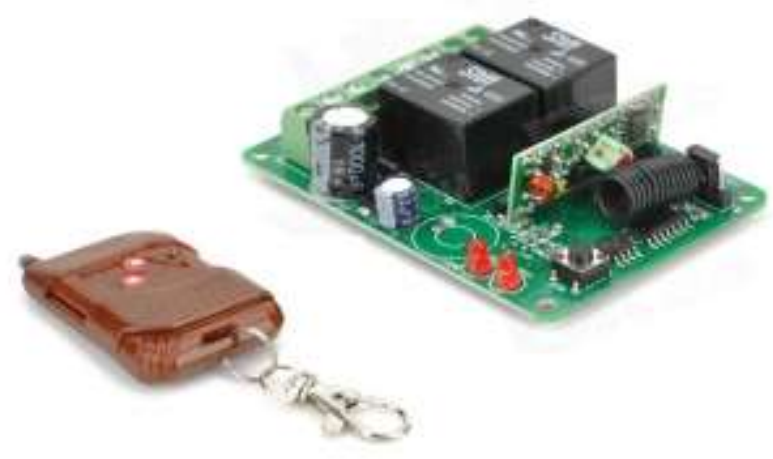

Figure 9. RF transmitter and receiver

\section{Results and Discussion}

Ultrasonic sensors, infrared sensor, PIC 18F46K80 and ISD1932 are tested individually as well as integrated. As ultrasonic sensors work on principle of echo, studying of its reflection on different obstacle is very important.

The measurement cycle starts with microcontroller transmitting the $10 \mu \mathrm{s}$ high level pulse to the sensor trigger pin to start ranging (T1), then the sensor will send out ultrasonic signal with $40 \mathrm{kHz}$ and $450 \mu \mathrm{s}$ (T2) and then wait to capture the rising edge output by echo port (T3) from $150 \mu \mathrm{s}: 25 \mathrm{~ms}$, depending on measured distance as shown in Fig. 10. In case of no obstacle (no signal reflected) it waits $38 \mathrm{~ms}$ before it restarts transmission.

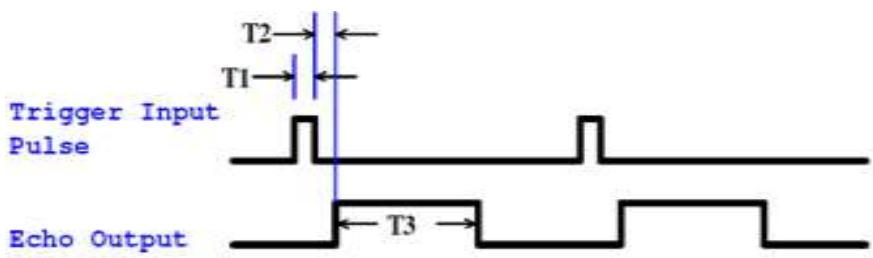

Pulse

Figure 10. Timing diagram

Ultrasonic distance sensor uses time of flight (TOF) to detect obstacle - the output is a digital pulse which length is the time it takes for the sound to reach the target and return.
Several experiments were done on obstacles at different distance and the average TOF results are shown in Fig. 11.

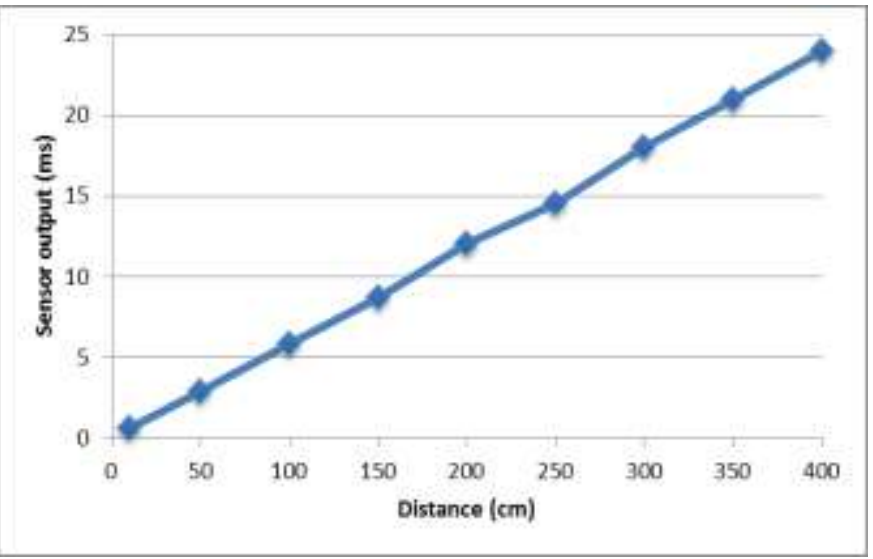

Figure 11. Time of flight vs measured distance of ultrasonic sensor

TABLE II. RESUlT OF ULTRASONIC SENSOR COMPARISON

\begin{tabular}{|c|c|c|c|}
\hline Distance (cm) & $\begin{array}{c}\text { Analog value } \\
\text { calculated (mV) }\end{array}$ & $\begin{array}{c}\text { Analog value } \\
\text { measured (mV) }\end{array}$ & error \\
\hline 5 & 25 & 24 & $1 \mathrm{mv}$ \\
\hline 10 & 50 & 48.8 & $1.8 \mathrm{mv}$ \\
\hline 20 & 100 & 97.6 & $2.4 \mathrm{mv}$ \\
\hline 30 & 150 & 146.4 & $3.6 \mathrm{mv}$ \\
\hline 40 & 200 & 195.3 & $4.7 \mathrm{mv}$ \\
\hline 50 & 250 & 244.15 & $5.85 \mathrm{mv}$ \\
\hline 75 & 375 & 366 & $9 \mathrm{mv}$ \\
\hline 100 & 500 & 489 & $11 \mathrm{mv}$ \\
\hline 150 & 750 & 732 & $16 \mathrm{mv}$ \\
\hline 200 & 1000 & 976.6 & $23.4 \mathrm{mv}$ \\
\hline 250 & 1250 & 1220.7 & $29.3 \mathrm{mv}$ \\
\hline 300 & 1500 & 1464.9 & $35.1 \mathrm{mv}$ \\
\hline 350 & 1750 & 1709 & $41 \mathrm{mv}$ \\
\hline 400 & 2000 & 1953.2 & $46.8 \mathrm{mv}$ \\
\hline
\end{tabular}

We tested how the ultrasonic sensors performance in lab compared to simulated calculation. TABLE II and Fig. 12 are present comparison of the ultrasonic sensor analog voltage value between the calculation value and measurement value. Thereafter the error is calculated in small range $5: 50 \mathrm{~cm}$ error is $1-6 \mathrm{mv}$, medium range $75: 200 \mathrm{~cm}$ error is $9-23 \mathrm{mv}$ and far range $250: 400 \mathrm{~cm}$ error is $30-47 \mathrm{mv}$.

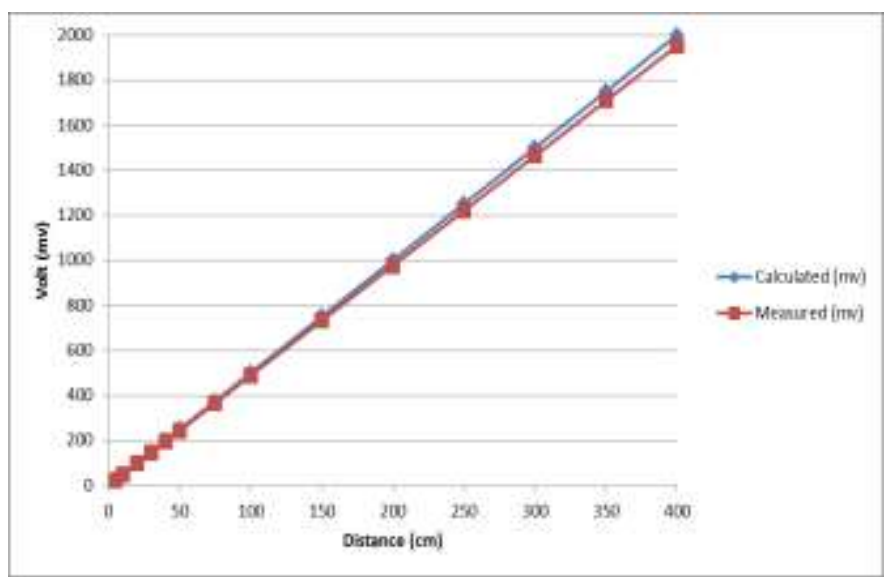

Figure 12. Difference between calculated and measured value 
Proc. of the Second Intl. Conf. on Advances in Bio-Informatics and Environmental Engineering - ICABEE 2015

Copyright $\odot$ Institute of Research Engineers and Doctors, USA .All rights reserved.

ISBN: 978-1-63248-043-9 doi: 10.15224/ 978-1-63248-043-9-29

TABLE III. COMPARISON OF OTHER DEVICES

\begin{tabular}{|c|c|c|c|c|}
\hline Devices & Detection Range & Time Response & Power Consumption & $\begin{array}{c}\text { Stairs \& Water } \\
\text { Detection }\end{array}$ \\
\hline Proposed System & High & Fast & Low & Yes \\
\hline $\begin{array}{c}\text { Vibration and Voice Operated Navigation } \\
\text { System for Visually Impaired Person }\end{array}$ & Low & medium & Low & Yes \\
\hline $\begin{array}{c}\text { A Smart Infrared Microcontroller-Based Blind } \\
\text { Guidance System }\end{array}$ & Medium & Fast & Low & yes \\
\hline $\begin{array}{c}\text { Ultrasonic Spectacles and Waist-belt for } \\
\text { Visually Impaired and Blind Person }\end{array}$ & High & Fast & Low & No \\
\hline
\end{tabular}

To evaluate the performance of this stick, testing is performed in real world, by actual beneficiaries (trained and untrained) blind people. Two experiments have been carried out using a number of obstacles. The experiment was conducted by six blind people from which two were trained on using the stick and four were not.

During experiment, a blind person was asked to walk through the testing area where different type of obstacles has been placed within 15 meter range. The user's walking speed is recorded. Time taken by trained and untrained for successfully walking through the obstacles is measured as shown in Fig. 13. Then we calculated the average speed of trained users to be $0.80 \mathrm{~m} / \mathrm{s}$ and untrained users to be $0.41 \mathrm{~m} / \mathrm{s}$. In comparison with the traveling speed of the sighted people be $(1.4 \mathrm{~m} / \mathrm{s})$. This result shows that training of the user reflected in gaining twice traveling speed and also increased the user trust in avoiding obstacles in free path.

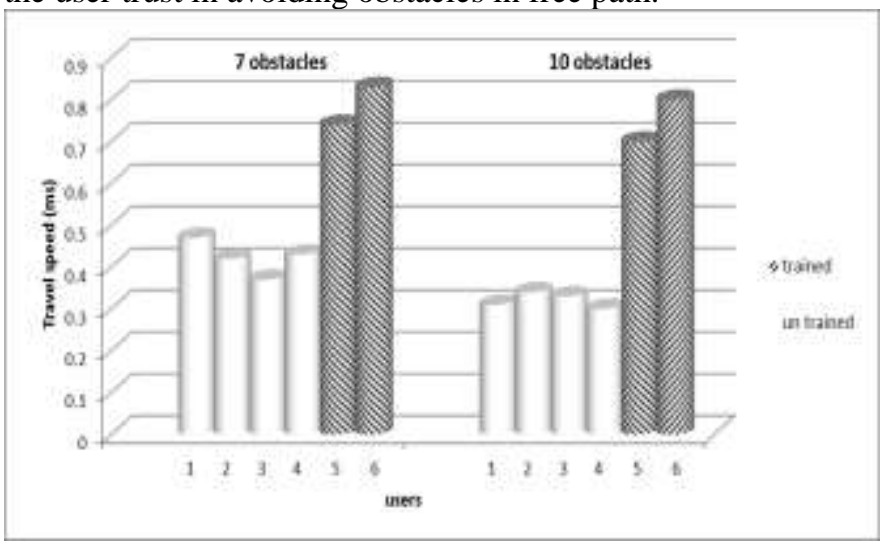

Figure 13. Performance and accuracy of the smart stick

The proposed system should be compared with available and up to date technology. Several parameters should be considered to evaluate the performance of the proposed guidance system such as the range of detection, time response and power consumption.
- The first parameter is the range of detection. A tool which can find obstacles throughout $0-2 \mathrm{~m}$ can be considered as a low range device, $2-4 \mathrm{~m}$ as medium range, while higher than $4 \mathrm{~m}$ is considered as high range.

- The second parameter is the response time, and a system sensing and responding $0-100 \mathrm{~ms}$ is regarded as fast, $100-200 \mathrm{~ms}$ medium and higher than $200 \mathrm{~ms}$ as slow.

- The third important parameter is the power consumption of the system and how long it will stay working without the need to recharge. The following ratings are considered: consumption of an electrical power of $00.5 \mathrm{~W}$ is regarded as low power, $0.5-1 \mathrm{~W}$ as medium consumption, and higher than $1 \mathrm{~W}$ as high consumption.

- The device is portable if it is light in weight and the user can easily wear for extended period of time otherwise it is considered non-portable.

We compared our performance with last 5 years literature with 3 devices. Vibration and Voice Operated Navigation System for Visually Impaired Person [17] consists of three ultrasonic sensors, PIC microcontroller16F877A using the feedback through vibration and voice alerts the user if any obstacle is around and within $70 \mathrm{~cm}$, A Smart Infrared Microcontroller-Based Blind Guidance System [18] consists of three infrared sensor, microcontroller 16F877A using feedback through vibration and tune speakers warn the user if any obstacles is around within $2 \mathrm{~m}$, Ultrasonic Spectacles and Waist-belt for Visually Impaired and Blind Person [21] consists of five ultrasonic sensors, APR9600 audio recording and playback flash memory, earphone with AT89S52 microcontroller. The comparison is shown in TABLE III. 


\section{Conclusion}

The Smart Stick acts as a basic platform for the coming generation of more aiding devices to help the visually impaired to be more safe. It is effective and afford. It leads to good results in detecting the obstacles lying ahead of the user in a range of four meters, detecting stairs and water pits.

This system offers a low-cost, reliable, portable, lowpower consumption and robust solution for navigation with obvious short response time. Though the system is hard-wired with sensors and other components, it's light in weight. Further aspects of this system can be improved via wireless connectivity between the system components, thus, increasing the range of the ultrasonic sensor and implementing a technology for determining the speed of approaching obstacles. While developing such an empowering solution, visually impaired and blind people in all developing countries were on top of our priorities.

\section{Acknowledgment}

This research was supported by Academy of Scientific Research and Technology (ASRT), through the funding of the electronics research Institute (ERI) through support the implementation of innovative initiatives to contribute to solving some of the problems of society.

\section{References}

[1] World Health Organization, "Visual Impairment and Blindness," Fact sheet N “282”, Oct 2014.

[2] National Disability Policy: A Progress Report - October 2014, National Council on Disability, Oct 2014.

[3] T. Terlau and W. M. Penrod, "K'Sonar Curriculum Handbook", Available from: "http://www.aph.org/manuals/ksonar.pdf", June 2008

[4] L. Whitney, "Smart cane to help blind navigate", Available from: "http://news.cnet.com/8301-17938\_105-10302499-1.html", 2009.

[5] J.M. Hans du Buf, J.Barroso, Jojo M.F. Rodrigues, H.Paredes, M.Farrajota, H.Fernandes, J.Jos, V.Teixeira, M.Saleiro.’The SmartVision Navigation Prototype for Blind Users". International Journal of Digital Content Technology and its Applications, Vol.5 No .5, pp. 351 - 361, May 2011.

[6] I. Ulrich, and J. Borenstein, "The guide cane-Applying mobile robot technologies to assist the visually impaired," IEEE Transaction on Systems, Man, and Cybernetics-Part A: Systems and Humans, vol. 31, no. 2, pp. 131-136, 2001.

[7] P. Meijer, "An Experimental System for Auditory Image Representations," IEEE Transactions on Biomedical Engineering, vol.39, no 2, pp. 112-121, Feb 1992.

[8] M. Nie, J. Ren, Z. Li et al., "SoundView: an auditory guidance system based on environment understanding for the visually impaired people," in Proceedings of the 31st Annual International Conference of the IEEE Engineering in Medicine and Biology Society: Engineering the Future of Biomedicine (EMBC '09), pp.7240-7243, IEEE, September 2009.

[9] G. Balakrishnan, G. Sainarayanan, R. Nagarajan and S. Yaacob, "Wearable Real-Time Stereo Vision for the Visually Impaired," Engineering Letters, vol. 14, no. 2, 2007.

[10] G. P. Fajarnes, L. Dunai, V. S. Praderas and I. Dunai, "CASBLiP- a new cognitive object detection and orientation system for impaired people," Proceedings of the 4th International Conference on Cognitive Systems, ETH Zurich, Switzerland, 2010.
[11] B.Hoyle, D.Withington, D.Waters, "UltraCane", Available from: "http://www.soundforesight.co.uk/index.html"". June 2006.

[12] E. Kee, "iSONIC cane for the virtually impaired", Available from: "http://www.ubergizmo.com/2011/01/isonic-cane-for-the-virtuallyimpaired/", 2011.

[13] R.Farcy, R.Leroux, R.Damaschini, R.Legras, Y.Bellik, C.Jacquet, J.Greene, P.Pardo, "Laser Telemetry to improve the mobility of blind people: report of the 6 month training course", ICOST $20031^{\text {st }}$ International Conference On Smart homes and health Telematics" Independent living for persons with disabilities and elderly people, Paris, pp. 24-26, 2003.

[14] Amit kumar, M. Manjunatha and J. Mukhopadhyay, "An Electronic Travel Aid for Navigation of Visually Impaired Person," Proceeding of the 3rd International Conference on Communication Systems and Networks, pp.1-5, 2011.

[15] Bouhamed, Sonda Ammar, Imene Khanfir Kallel, and Dorra Sellami Masmoudi. "New electronic white cane for stair case detection and recognition using ultrasonic sensor." International Journal of Advanced Computer Science \& Applications 4.6, 2013.

[16] Design of non-weighing type desert plant lysimeter observation system based on PIC18. In Information Management, Innovation Management and Industrial Engineering (ICIII), 6th International Conference on IEEE,Vol. 3, pp. 42-44, 2013.

[17] N.Mahmud, R.K.Saha, R.B. Zafar, M.B.H. Bhuian, and S.S.Sarwar, "Vibration and Voice Operated Navigation System forVisually Impaired Person," In Informatics, Electronics \& Vision (ICIEV), International Conference on IEEE, pp. 1-5, 2014.

[18] Amjed S. Al-Fahoum, Heba B. Al-Hmoud, and Ausaila A. Al-Fraihat, "A Smart Infrared Microcontroller-Based Blind Guidance System". Active and Passive Electronic Components, 2013.

[19] Su, B., \& Wang, L.“Application of Proteus virtual system modelling (VSM) in teaching of microcontroller". In E-Health Networking, Digital Ecosystems and Technologies (EDT), 2010 International Conference on Vol. 2, pp. 375-378, 2010.

[20] Direct mode ISD 1932. (2009, January 5) [Online]. https://www.futurashop.it/...PDF_ENG/7300-ISD1932.pdf.

[21] Bhatlawande, Shripad S., Jayant Mukhopadhyay, and Manjunatha Mahadevappa. "Ultrasonic spectacles and waist-belt for visually impaired and blind person." Communications (NCC), National Conference on. IEEE, 2012. 